\title{
Flrt2 is involved in fine-tuning of osteoclast multinucleation
}

\author{
Jumpei Shirakawa ${ }^{1}$, Noriko Takegahara ${ }^{1}$, Hyunsoo $\mathrm{Kim}^{1}$, Seoung Hoon Lee ${ }^{1,2}$, Kohji Sato $^{3}$, Satoru Yamagishi ${ }^{3} \mathcal{E}^{\circ}$ \\ Yongwon $\mathrm{Choi}^{1, *}$ \\ ${ }^{1}$ Department of Pathology and Laboratory Medicine, University of Pennsylvania Perelman School of Medicine, Philadelphia, PA 19104, \\ USA, ${ }^{2}$ Department of Oral Microbiology and Immunology, College of Dentistry, Wonkwang University, Iksan 54538, Korea, ${ }^{3}$ Department \\ of Organ and Tissue Anatomy, Hamamatsu University School of Medicine, Hamamatsu, Shizuoka 431-3192, Japan
}

\begin{abstract}
Osteoclasts are multinucleated giant cells derived from myeloid progenitors. Excessive bone resorption by osteoclasts can result in serious clinical outcomes for which better treatment options are needed. Here, we identified fibronectin leucine-rich transmembrane protein 2 (Flrt2), a ligand of the Unc5 receptor family for neurons, as a novel target associated with the late/maturation stage of osteoclast differentiation. Flrt2 expression is induced by stimulation with receptor activator of nuclear factor-kB ligand (RANKL). Flrt2 deficiency in osteoclasts results in reduced hyper-multinucleation, which could be restored by RNAi-mediated knockdown of Unc5b. Treatment with Netrin1, another ligand of Unc5b which negatively controls osteoclast multinucleation through down regulation of RANKL-induced Rac1 activation, showed no inhibitory effects on Flrt2-deficient cells. In addition, RANKLinduced Rac1 activation was attenuated in Flrt2-deficient cells. Taken together, these results suggest that FIrt2 regulates osteoclast multinucleation by interfering with Netrin 1-Unc5b interaction and may be a suitable therapeutic target for diseases associated with bone remodeling. [BMB Reports 2019; 52(8): 514-519]
\end{abstract}

\section{INTRODUCTION}

Skeletal homeostasis is maintained by balancing the bone forming function of osteoblasts and bone resorbing function of osteoclasts. Functional imbalance of osteoblasts and osteoclasts results in abnormal bone homeostasis (1). Osteoclasts are specialized multinucleated giant cells derived from monocyte/ macrophage lineage bone marrow precursors (2). Osteoclast differentiation is initiated and sustained primarily by the

*Corresponding author. Tel: +1-215-746-6404; Fax: +1-215-5730888; E-mail: ychoi3@pennmedicine.upenn.edu

https://doi.org/10.5483/BMBRep.2019.52.8.116

Received 24 April 2019, Revised 12 May 2019, Accepted 15 June 2019

Keywords: FLRT2, Hyper-multinucleation, Netrin 1, Osteoclast, Rac1, UNC5b osteoclast differentiation factor RANKL, which is mainly produced by osteoblasts and osteocytes (3, 4). Under pathogenic inflammatory conditions, excessive bone resorption by osteoclasts is often observed $(5,6)$. Currently, bisphosphonate and anti-RANKL antibody (Denosumab), which target early osteoclast commitment and/or osteoclast viability, are used for treatment (7). Although these treatments are efficiently anti-resorptive, long-term use compromises bone strength due to unintended inhibition of coupled bone formation. A better treatment strategy may be to target only late-stage osteoclast biological processes (i.e., osteoclast "maturation"), while preserving early osteoclast differentiation necessary for coupled bone formation (8). To do so in a manner that could lead to novel effective therapeutics to target bone remodeling diseases will require further investigation of the molecular mechanisms that control osteoclast maturation.

A hallmark of osteoclast maturation is multinucleation (9). Multinucleation is required for physiological bone resorbing activities (10), but hyper-multinucleation (defined by osteoclasts with $>100$ micron diameter) often results in pathological bone loss $(5,6,11)$. To identify gene targets associated with the late/maturation stage of osteoclast differentiation, we have previously performed comparative gene expression profiling by using multinucleation as a functional readout (8). As a result, we identified the gene Flrt 2 which encodes the protein fibronectin leucine-rich transmembrane protein 2 (Flrt2) (Supplementary Fig. 1). Flrt2 is a member of the fibronectin leucine-rich transmembrane family of proteins (Flrts: Flrt1-3) (12), which are classified as cell adhesion molecules (CAMs) (13), and are also reported to interact with heterophilic receptors, functioning as guidance factors in vascular, neural, and early embryonic development (14-21). Flrt2 has been reported to function as a chemorepellent in axon guidance and cell migration through interactions with uncoordinated-5 (Unc5) receptors (19), and also to function in cell adhesion through an interaction with Latrophilins (22).

In this study, we identified Flrt2, which has no previously reported function in bone, as a regulator of osteoclast multinucleation. We report here that gene deletion of Flrt2 in osteoclasts resulted in reduced osteoclast hyper-multinucleation. RNAi-mediated knockdown of Unc5b in Flrt2-deficient cells restored osteoclast hyper-multinucleation. Treatment with 
Netrin1, an alternative ligand of Unc5b which has been shown to inhibit osteoclast multinucleation through negative regulation of RANKL-induced Rac1 activation (23), showed no inhibitory effects on Flrt2-deficient cultures. Additionally, RANKLinduced Rac1 activation was impaired in Flrt2-deficient osteoclasts. These results suggest the possibility that Flrt2 and Netrin 1 compete for Unc5b binding during osteoclast multinucleation, as osteoclast hyper-multinucleation is inhibited in Flrt2-deficient cells. Collectively, our results reveal a novel role for Flrt2 in fine-tuning of osteoclast multinucleation.

\section{RESULTS}

Flrt2 is involved in in vitro osteoclast differentiation

In order to understand the role of Flrt2 in osteoclasts, we first examined Flrt2 gene expression dynamics during osteoclast differentiation. We generated osteoclasts in vitro from mouse bone marrow-derived monocytes (BMMs) treated with M-CSF + RANKL for up to three days, and performed temporal qPCR expression analysis and western blots using whole cell lysates pulled down with a lectin that recognize glycoproteins including Flrt2. Expression of Flrt2 was induced, and peaked on day one after RANKL stimulation (Fig. 1A). We next generated Flrt2 conditional knockout mice by crossing floxed Flrt2 mice (Flrt2 ${ }^{\mathrm{fl} / \mathrm{fl}}$ ) with Mx1Cre mice, which express inducible Cre recombinase, since global deletion of Flrt2 expression in mice leads to embryonic lethality (17). In Flrt $2^{\mathrm{fl} / \mathrm{fl}} \mathrm{Mx} 1 \mathrm{Cre}$ mice, the Flrt2 gene is deleted upon polyinosinic-polycytidylic acid (poly I:C) treatment in osteoclast precursors, enabling us to examine the effect of Flrt2 deletion on osteoclast differentiation. We generated BMMs from Flrt2 ${ }^{\mathrm{fl} / \mathrm{ll}}$, Flrt2 ${ }^{\mathrm{fl} /+} \mathrm{Mx} 1 \mathrm{Cre}$, and Flrt2 ${ }^{\mathrm{fl} / \mathrm{fl}} \mathrm{Mx} 1 \mathrm{Cre}$ mice, and confirmed Flrt2 gene deletion in genomic DNA from Flrt2 ${ }^{\mathrm{fl} / \mathrm{fl}} \mathrm{Mx} 1 \mathrm{Cre}$ BMMs using PCR (Fig. 1B). Consistent with this, Flrt2 gene expression was completely abolished in Flrt2 ${ }^{\mathrm{f} / \mathrm{fl}} \mathrm{Mx} 1 \mathrm{Cre} \mathrm{BMMs}$ (Fig. 1B). We then examined whether Flrt2 gene deletion affects osteoclast formation. We prepared Flrt $2^{\mathrm{fl} / f \mathrm{l}}$ and Flrt $2^{\mathrm{fl} / f \mathrm{l}} \mathrm{M} \times 1$ Cre BMMs and cultured with M-CSF + RANKL to generate osteoclasts. We found reductions in total tartrate-resistant acidic phosphatase (TRAP) activity and in frequency of multinucleated TRAP ${ }^{+}$ cells (i.e., mature osteoclasts) in Flrt $2^{\mathrm{fl} / \mathrm{fl}} \mathrm{Mx} 1 \mathrm{Cre}$ cultures compared to Flrt $2^{\mathrm{f} / f / \mathrm{l}}$ cultures (Fig. 1C). Reductions in hyper-multinucleated osteoclasts $(>100 \mu \mathrm{m})$ were particularly dramatic (Fig. 1C). Message levels for the osteoclast differentiation and maturation markers Nfatc1, CtsK, and Atp6v0d2 showed small but still significant differences in Flrt2 $2^{\text {fl/fl }} \mathrm{Mx} 1 \mathrm{Cre}$ cultures on Day 3, while Dcstamp showed no differences (Fig. 1D). Retroviral transduction of Flrt $2^{\mathrm{fl} / \mathrm{fl}} \mathrm{M} \times 1 \mathrm{Cre} \mathrm{BMMs}$ with full length Flrt2 completely restored TRAP $^{+}$hyper-multinucleated cells in Flrt $2^{\mathrm{fl} / \mathrm{fl}} \mathrm{M} \times 1$ Cre cultures (Fig. 1E), suggesting that the phenotypes observed in Flrt2 ${ }^{\mathrm{f} / \mathrm{fl}} \mathrm{M} \mathrm{M} 1 \mathrm{Cre}$ cultures can be attributed to deletion of the Flrt2 gene. These results suggest a role for osteoclast-derived FIrt2 in osteoclast differentiation, and more specifically in osteoclast hyper-multinucleation.

\section{Osteoclast-derived Flrt2 is required for bone homeostasis}

To examine whether osteoclast-derived Flrt2 is required for bone development and homeostasis, we performed representative $3 \mathrm{D}$ reconstructions of trabecular bone from the femurs of gender- and age-matched Flrt2 $2^{\mathrm{fl} / \mathrm{fl}}$ and Flrt2 $2^{\mathrm{fl} / \mathrm{fl}} \mathrm{Mx} 1 \mathrm{Cre}$ mice. Bone microstructure imaging by high resolution microcomputed tomography $(\mu \mathrm{CT})$ of Flrt $2^{\mathrm{f} / \mathrm{fl}} \mathrm{M} \times 1$ Cre mice revealed slight but significant increases in bone mass, characterized by

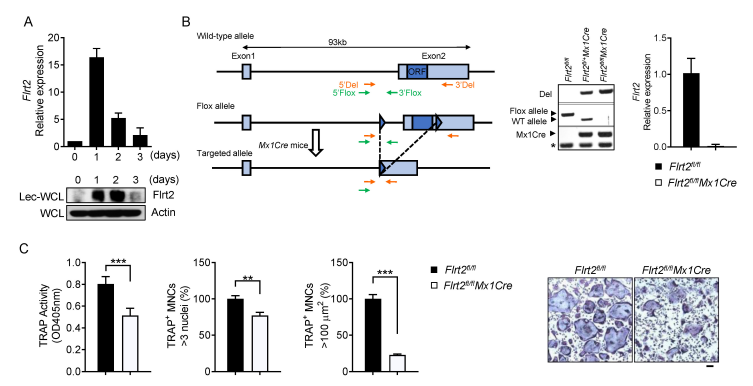

D
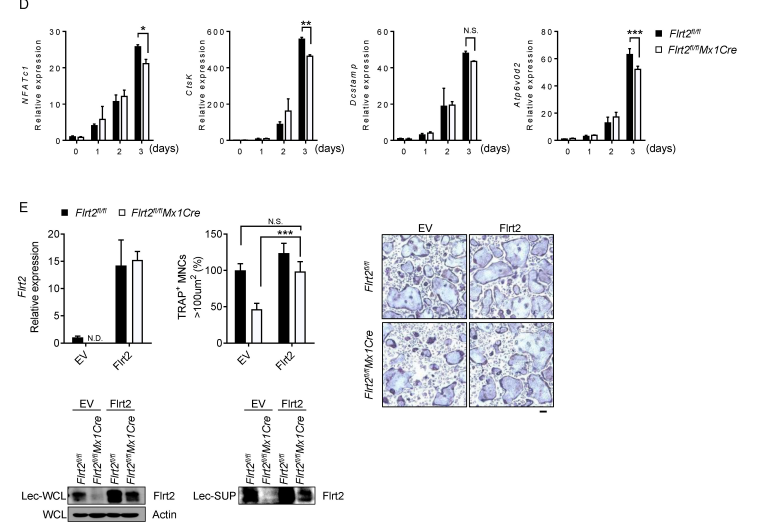

Fig. 1. Flrt2 is involved in in vitro osteoclast differentiation. (A) Expression of Flrt2 was quantified by Q-PCR (top) and western blot with glycoprotein-enriched whole cell lysates (lec-WCL) (bottom). (B) Scheme of the Flrt2 knock-out allele (left). PCR genotyping of Flrt2 $2^{\mathrm{fl} / \mathrm{fl}}$, Flrt2 ${ }^{\mathrm{fl} / \mathrm{+}} \mathrm{Mx} 1 \mathrm{Cre}$, and Flrt2 $2^{\mathrm{fl} / \mathrm{fl}} \mathrm{M} \times 1 \mathrm{Cre} \mathrm{BMMs}$ Flox allele and targeted allele were detected by genomic PCR with Flox primers (green arrows in left) and Del primers (orange arrows in left), respectively. Cre recombinase was detected by genomic PCR. Asterisk indicates internal controls (middle). Flrt2 expression in $\mathrm{Flrt}^{\mathrm{fl} / \mathrm{fl}}$ and Flrt2 $2^{\mathrm{fl} / \mathrm{fl}} \mathrm{Mx} 1 \mathrm{Cre} \mathrm{BMMs}$ (right). (C) Flrt2 ${ }^{\mathrm{f} / / \mathrm{fl}}$ and Flrt2 ${ }^{\mathrm{f} / \mathrm{fl}} \mathrm{M} \times 1 \mathrm{Cre}$ BMMs were cultured with M-CSF + RANKL for three days. TRAP activity, frequency of TRAP $^{+}$multinucleated cells (3 nuclei or more per cell), and frequency of TRAP hyper-multinucleated cells $\left(>100 \mu^{2}\right)$ are shown. (D) Expression of indicated genes was quantified by Q-PCR. (E) $\mathrm{Flrt}^{\mathrm{fl} / \mathrm{fl}}$ and $\mathrm{Flrt} 2^{\mathrm{fl} / \mathrm{fl}} \mathrm{M} \times 1 \mathrm{Cre} \mathrm{BMMs}$ were retrovirally transduced with the indicated vectors followed by culture with M-CSF + RANKL for three days. Overexpression of Flrt2 was confirmed by Q-PCR (Day 3 after RANKL) and western blot with lec-WCL and glycoprotein-enriched culture supernatants (lec-SUP) (Day 2 after RANKL). Frequency of $\mathrm{TRAP}^{+}$hyper-multinucleated cells $(>100$ $\mu \mathrm{m}^{2}$ ) is shown. Scale bars represents $100 \mu \mathrm{m}$. Data are shown as mean \pm S.D. ${ }^{*} \mathrm{P}<0.05, * * \mathrm{P}<0.01, * * * \mathrm{P}<0.001$. 
augmented bone indices including trabecular bone volume per tissue volume (BV/TV), trabecular number (Tb.N), and concomitant decreases in trabecular spacing (Tb.Sp) (Fig. 2). No significant differences in trabecular thickness or cortical bone thickness (Ct.Th) were detected between Flrt2 ${ }^{\mathrm{fl} / \mathrm{fl}}$ and Flrt $2^{\mathrm{fl} / \mathrm{fl}} \mathrm{M} \times 1 \mathrm{Cre}$ mice (Fig. 2). These results suggest that osteoclast-expressed Flrt2 is involved in bone homeostasis.

\section{Flrt2, Netrin 1 and Unc5b regulation of osteoclast multinucleation}

To understand the molecular mechanism that regulates Flrt2-mediated osteoclast hyper-multinucleation, we examined RANKL-dependent signaling pathways, including ERK, p38, JNK, and NF-kB, which are activated via the RANK-TRAF6 axis. We found no significant differences in activation of these signaling molecules between Flrt2 ${ }^{\mathrm{fl} / \mathrm{fl}}$ and Flrt2 ${ }^{\mathrm{fl} / \mathrm{fl}} \mathrm{Mx} 1 \mathrm{Cre}$ cultures (data not shown), suggesting that osteoclast-expressing Flrt2 is not involved in regulation of the RANK-TRAF6 signaling axis.

Flrts have been reported to act as CAMs and also as ligands of repulsive receptors $(21,24)$. Flrts have also been reported to be proteolytically cleaved near the plasma membrane in neurons (19). To determine whether Flrt2 functions as a CAM or as a ligand in osteoclasts, we examined cleavage of Flrt2 during osteoclast differentiation. We prepared whole cell lysates and supernatants from $\mathrm{Flrt} 2^{\mathrm{fl} / f \mathrm{ll}}$ and $\mathrm{Flrt} 2^{\mathrm{fl} / \mathrm{fl}} \mathrm{M} \times 1 \mathrm{Cre}$ cultures, pulled down with a lectin, and performed western blotting using Flrt2-specific antibody. Membrane-bound Flrt2, which ranges in size from 80 to $100 \mathrm{kDa}$, was detected in whole cell lysate of Flrt2 ${ }^{\mathrm{fl} / \mathrm{fl}}$ cultures one day after RANKL stimulation (Fig. 3A). Expression of membrane-bound Flrt2 gradually decreased during osteoclast differentiation, and disappeared almost completely in mature osteoclast cultures. By contrast, the cleaved form of Flrt2, which ranges in size from 60 to $80 \mathrm{kDa}$, was detected in supernatants from two days after RANKL stimulation (Fig. 3A). The cleaved form of Flrt2 was decreased after treatment with Brefeldin A, a trafficking inhibitor, suggesting that trafficking of membranebound Flrt2 was inhibited, and consequently, that presence of the cleaved form was decreased (Supplementary Fig. 2).
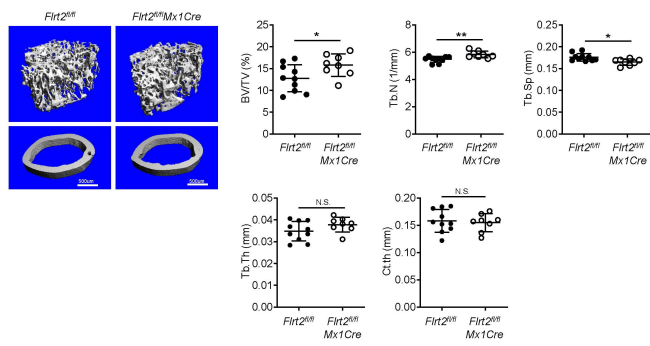

Fig. 2. Osteoclast-derived Flrt2 is required for bone homeostasis. $\mu \mathrm{CT}$ images of femurs from Flrt2 $2^{\mathrm{f} / \mathrm{fl}}$ and Flrt $2^{\mathrm{fl} / \mathrm{fl}} \mathrm{Mx} 1 \mathrm{Cre}$ mice. BV/TV, Tb.Th, Tb.N, Tb.Sp, and Ct.Th are shown. Scale bars represent 0.5 $\mathrm{mm}$. Data are shown as mean \pm S.D. $* P<0.05$, $* * P<0.01$.
Neither membrane-bound nor cleaved forms of Flrt2 were detected in Flrt $2^{\mathrm{fl} / \mathrm{fl}} \mathrm{Mx} 1 \mathrm{Cre}$ cultures (Fig. 3A). The cleaved form of Flrt2 was detected in Flrt $2^{\mathrm{fl} / \mathrm{fl}} \mathrm{Mx} 1 \mathrm{Cre}$ cultures after retroviral transduction of Flrt2 (Fig. 1E). These results suggest that osteoclast-expressed Flrt2 is cleaved during osteoclast differentiation, and further suggest that Flrt2 might predo-

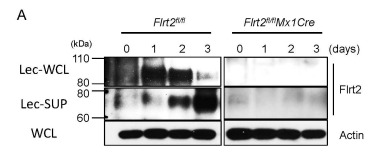

B
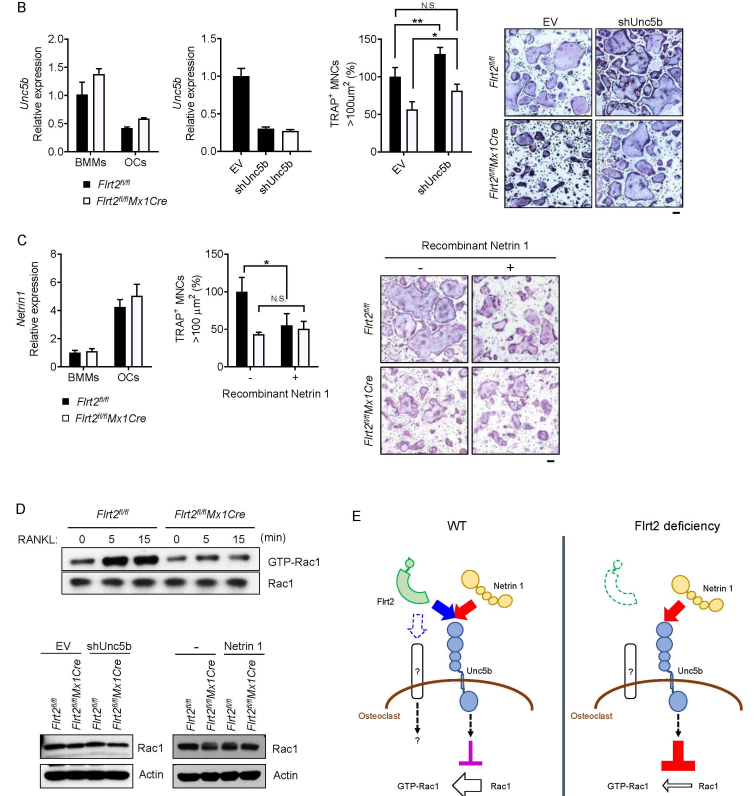

Fig. 3. Flrt2, Netrin 1 and Unc5b regulation of osteoclast multinucleation. (A) Western blot analysis of lec-WCL and lec-SUP was performed using anti-Flrt2 antibody. (B) Expression of Unc5b message was quantified by Q-PCR (left). Flrt2 ${ }^{\text {fl/fl }}$ and Flrt2 $2^{\mathrm{fl} / \mathrm{P}} \mathrm{M} \times 1 \mathrm{Cre} \mathrm{BMMs}$ retrovirally transduced with shUnc5b or control vector were cultured with M-CSF + RANKL for three days, and knockdown of Unc5b was determined by Q-PCR (middle). Frequency of TRAP $^{+}$hyper-multinucleated cells is shown (right). (C) Expression of Netrin 1 message was quantified by Q-PCR (left). Flrt2 ${ }^{\mathrm{f} / \mathrm{fl}}$ and Flrt2 ${ }^{\mathrm{f} / \mathrm{fl} / \mathrm{M}} 1 \mathrm{Cre} \mathrm{BMMs}$ were cultured with M-CSF + RANKL for three days in the presence or absence of recombinant Netrin $1(200 \mathrm{ng} / \mathrm{ml})$. Frequency of TRAP $^{+}$hypermultinucleated cells is shown (right). (D) Pre-osteoclasts from $\mathrm{Flrt} 2^{\mathrm{fl} / f \mathrm{l}}$ and $\mathrm{Flrt} 2^{\mathrm{f} / / \mathrm{fl}} \mathrm{M} \times 1 \mathrm{Cre}$ mice were stimulated with RANKL for the indicated times, and the activated form of Rac1 was detected (top). Rac1 protein levels in Unc5b-knockdown or Netrin 1-treated cultures were shown (bottom). (E) Schematic model of regulation of osteoclast multinucleation by Flrt2. Osteoclast-derived Flrt2 competes with osteoclast-derived Netrin 1 for a receptor Unc5b, which attenuates negative regulation of Netrin 1-Unc5b-mediated Rac1 activation. In the absence of osteoclast-derived Flrt2, Netrin $1-$ Unc5b interaction inhibited Rac1 activation, leading to impaired osteoclast multinucleation. Scale bars represents $100 \mu \mathrm{m}$. Data are shown as mean \pm S.D. ${ }^{*} P<0.05, * * P<0.01, * * * P<$ 0.001 . 
minantly function as a soluble ligand that interacts with heterophilic receptors, but that does not function as a CAM.

Flrt2 has been shown to interact with Unc5b, Unc5d and/or Latrophilin3 $(18-20,22)$. Unc5b has been demonstrated as the predominant receptor for Flrt2 in endothelial cells during vascular development (20). To gain insight into the predominant receptor for Flrt2 in osteoclast formation, we performed qPCR expression analysis. We detected expression of Unc5b (Fig. 3B) but not Unc5d or Latrophilin3 (data not shown). The expression levels of Unc5b were comparable between Flrt $2^{\mathrm{fl} / \mathrm{fl}} \mathrm{M} \times 1 \mathrm{Cre}$ and Flrt2 $2^{\mathrm{fl} / \mathrm{fl}}$ cultures, and decreased in osteoclasts (Fig. 3B). To identify the involvement of Unc5b in Flrt2-mediated regulation of osteoclast multinucleation, we performed RNAi experiments using retrovirus encoding shRNA for Unc5b. RNAi-mediated knockdown of Unc5b in $\mathrm{Flrt}^{\mathrm{fl} / \mathrm{fl}} \mathrm{Mx} 1 \mathrm{Cre} \mathrm{BMMs}$ restored osteoclast hyper-multinucleation, and furthermore, knockdown of Unc5b in Flrt $2^{\mathrm{flfl} / \mathrm{B}}$ BMMs resulted in slightly increased numbers of TRAP $^{+}$hypermultinucleated osteoclasts (Fig. 3B).

Unc5 receptors are known as classical signaling receptors for secreted Netrin ligands in the nervous system (24). In the bone system, Netrin 1 is produced by various bone cells, including osteoclasts $(23,25)$, and Netrin 1-Unc5b interactions negatively regulate osteoclast multinucleation (23). Interestingly, Netrin 1 has been shown to compete with Flrt2 for Unc5d (19). We found comparable message levels of Netrin 1 in both Flrt2 ${ }^{\mathrm{fl} / \mathrm{fl}}$ and Flrt2 $2^{\mathrm{fl} / \mathrm{fl}} \mathrm{Mx} 1 \mathrm{Cre} \mathrm{BMMs}$ and osteoclasts (Fig. 3C). We examined whether Netrin 1-Unc5b interactions mediated inhibitory effects on Flrt2-deficient osteoclast maturation. We prepared BMMs from Flrt2 ${ }^{\mathrm{fl} / \mathrm{fl}}$ and $\mathrm{Flrt} 2^{\mathrm{fl} / \mathrm{fl}} \mathrm{M} \times 1 \mathrm{Cre}$ mice and treated them with M-CSF and RANKL in the presence or absence recombinant Netrin 1. Formation of hyper-multinucleated osteoclasts was significantly inhibited in Flrt2/fl/l cultures, consistent with a previous report (Fig. 3C) (23). By contrast, an inhibitory effect of recombinant Netrin 1 was not observed in Flrt $2^{\mathrm{fl} / f \mathrm{l}} \mathrm{Mx} 1 \mathrm{Cre}$ cultures (Fig. 3C). These results suggested that Flrt2 might compete with Netrin 1 for Unc5b, such that Unc5b might be saturated with osteoclast-derived Netrin 1 in the absence of Flrt2, and consequently, no additional inhibition was observed in Flrt $2^{\mathrm{fl} / \mathrm{fl}} \mathrm{M} \times 1 \mathrm{Cre}$ cultures by adding recombinant Netrin 1.

Netrin 1-Unc5b interactions have been shown to inhibit RANKL-induced Rac1 activation (23). We therefore examined RANKL-induced Rac1 activation in Flrt2 $2^{\mathrm{fl} / \mathrm{fl}}$ and Flrt $2^{\mathrm{fl} / \mathrm{fl}} \mathrm{Mx} 1 \mathrm{Cre}$ cultures. We found that RANKL-induced Rac1 activation was diminished in Flrt2 ${ }^{\mathrm{fl} / \mathrm{fl}} \mathrm{M} \times 1 \mathrm{Cre}$ cultures compared to Flrt2 ${ }^{\mathrm{fl} / \mathrm{fl}}$ cultures (Fig. 3D). Rac1 protein levels were normal after RNAi-mediated knockdown of Unc5b and treatment with Netrin 1 in Flrt2 $2^{\mathrm{fl} / \mathrm{fl}}$ and Flrt $2^{\mathrm{fl} / \mathrm{fl}} \mathrm{M} \times 1 \mathrm{Cre}$ cultures (Fig. 3D). These results suggest that Flrt2 is required for proper RANKL-induced Rac1 activation, and further support, to some degree, the idea that osteoclast-derived Flrt2 might compete with Netrin 1 for Unc5b to attenuate the inhibitory effect of Netrin 1 on Rac1 activation. Collectively, our results suggest that osteoclast-derived Flrt2 competes with osteoclast-derived Netrin 1 for Unc5b binding, and contributes to fine-tuning of osteoclast multinucleation.

\section{DISCUSSION}

Osteoclast maturation involves multinucleation, which occurs via incomplete cytokinesis and/or cell fusion (26). The importance of multinucleation in osteoclast formation is demonstrated by the impaired bone-resorbing activity of osteoclasts that cannot become multinuclear cells (10). Although multinucleation is important for osteoclast function, hypermultinucleation is often observed in pathogenic inflammatory conditions where it contributes to excessive bone resorption. However, the molecular mechanisms underlying regulation of osteoclast hyper-multinucleation are not well understood. In this study, we identified Flrt2, which has previously been functionally characterized in systems other than bone, as a regulator for osteoclast multinucleation. Using conditional knockout mice, we revealed an osteoclast-intrinsic role of Flrt2 in multinucleation. Additionally, we showed involvement of Unc5b in Flrt2-mediated regulation of osteoclast multinucleation. Our results reveal Flrt2 as a novel regulator of osteoclast multinucleation.

We showed that expression of Flrt2 message peaked on Day 1 and gradually decreased (Fig. 1A). Retroviral transduction of Flrt2, which causes stable expression of Flrt2 restored hyper-multinucleation in $\mathrm{Flrt}^{\mathrm{ff/}} \mathrm{M} \times 1 \mathrm{Cre}$ cultures (Fig. 1E), suggesting that reduction of Flrt2 message during the maturation phase (Day 2 to 3 after RANKL stimulation) is not required for osteoclast maturation.

We here showed that osteoclast-expressed Flrt2 is cleaved during osteoclast differentiation, suggesting that osteoclastderived Flrt2 might function as a ligand, but not as a CAM. A previous report showed that Flrt2 cleavage requires metalloproteinase (MMP) activity (19). In osteoclasts, MMP-9 is the most abundant MMP (27), suggesting the possibility that MMP-9 is involved in Flrt2 cleavage in osteoclasts. Although we showed here that most osteoclast-expressed Flrt2 was cleaved in mature osteoclasts, we cannot conclude that membrane-bound Flrt2 does not play a role in osteoclasts, and further studies will be required to address this issue.

We sought to identify the molecular mechanisms by which Flrt2 regulates osteoclast multinucleation, and found that RNAi-mediated knockdown of Unc5b, the predominant receptor for Flrt2 in endothelial cells and also a receptor for Netrin 1 in osteoclasts, restored hyper-multinucleation in Flrt2 ${ }^{f / / f l} \mathrm{M} \times 1$ Cre cultures. These findings suggest that Unc5b is involved in Flrt2-mediated regulation of osteoclast hypermultinucleation. Given that Netrin 1 competes with Flrt2 for Unc5d (19), we hypothesized that Flrt2 could also compete with Netrin 1 for Unc5b, thus interfering with the Netrin 1-Unc5b-mediated negative regulation on osteoclast multinucleation (Fig. 3E). Indeed, we showed that 1) the inhibitory effect of Netrin 1 was not observed in Flrt2 ${ }^{\mathrm{fl} / \mathrm{fl}} \mathrm{Mx} 1 \mathrm{Cre}$ cultures, 
and 2) RANKL-induced Rac1 activation was inhibited in Flrt $2^{\mathrm{fl} / \mathrm{fl}} \mathrm{Mx} 1 \mathrm{Cre}$ cultures. Since message levels of Netrin 1 were not affected by the absence of Flrt2, it is plausible that reduced hyper-multinucleation in the absence of Flt2 is due in some part to negative regulation of Rac1 activation by Netrin 1 -Unc5b interactions. However, there is a difference between $\mathrm{Flrt}^{\mathrm{fl} / \mathrm{fl}}$ and Flrt2 ${ }^{\mathrm{fl} / \mathrm{fl}} \mathrm{Mx} 1 \mathrm{Cre}$ cultures in terms of frequency of hyper-multinucleated osteoclasts after RNAi-mediated knockdown of Unc5b (Fig. 3B), suggesting the involvement of a Unc5bindependent mechanism. Further studies will be required to determine the molecular mechanism underlying regulation of osteoclast multinucleation through Flrt2.

Considering the severity of the in vitro phenotype of Flrt2 ${ }^{\mathrm{fl} / \mathrm{fl} l} \mathrm{M} \times 1 \mathrm{Cre}$ osteoclast differentiation, the Flrt $2^{\mathrm{fl} / \mathrm{fl}} \mathrm{M} \times 1 \mathrm{Cre}$ bone phenotype seems modest. We found expression of another Flrt family member, Flrt3 (but not Flrt1), was induced in both Flrt2 $2^{\mathrm{fl} / \mathrm{fl}}$ and Flrt2 $2^{\mathrm{fl} / \mathrm{fl}} \mathrm{M} \times 1 \mathrm{Cre}$ cultures (Supplementary Fig. 3, and data not shown). Flrt3 has been demonstrated to act as a ligand of Unc5b in the nervous system. Indeed, RNAi-mediated knockdown of Flrt3 in Flrt2 $2^{\mathrm{flfl} / \mathrm{M}} \mathrm{M} 1 \mathrm{Cre}$ BMMs showed an additive inhibitory effect on osteoclast hypermultinucleation (Supplementary Fig. 3), raising the possibility of involvement of Flrt3 in osteoclast differentiation and bone homeostasis in vivo. Future characterization of Flrt2 and Flrt3 double conditional knockout mice may be useful for addressing this issue. In addition, Flrts are broadly expressed proteins. Given that Flrts are cleaved, cleaved Flrts derived from other tissues might compensate the lack of osteoclast-derived Flrt2 in vivo. Further studies will be required to clarify the impact of Flrts in bone homeostasis.

Taken together, although a full understanding of how Fltr2 contributes to osteoclast multinucleation and its impact on bone homeostasis remain unclear, we provide evidence of a fine-tuning function of the chemorepellent Flrt2 in osteoclast multinucleation. With additional characterization, Flrt2 may be a good candidate for a therapeutic target for bone diseases that are accompanied by bone destruction.

\section{MATERIALS AND METHODS}

\section{Mice}

Flrt $^{\mathrm{fl} / f l}$ mice were generated as described previously (20). To obtain Flrt2 conditional knock-out mice (Flrt2 ${ }^{\mathrm{fl} / \mathrm{fl}} \mathrm{Mx} 1 \mathrm{Cre}$ ) in hematopoietic cells, homozygous Flrt2 ${ }^{\mathrm{fl} / \mathrm{fl}}$ mice were crossed with $M \times 1 C r e$ transgenic mice $[\operatorname{Tg}(\mathrm{M} \times 1$-cre $) 1 \mathrm{Cgn}]$ purchased from Jackson Laboratory. For induction of Cre recombinase expression in $\mathrm{Mx} 1 \mathrm{Cre}$ mouse, mice were injected intraperitoneally with $250 \mu \mathrm{g}$ Poly(l:C) (SIGMA) 10 days after birth three times every other day. Mice were used for in vitro and in vivo experiments 6 weeks after the first injection. All mice were maintained and used in accordance with guidelines approved by the Institutional Animal Care and Use Committee (IACUC) at the University of Pennsylvania.

\section{Reverse transcription and real-time PCR (qPCR)}

Total RNAs were extracted from cells using Trizol reagent (Invitrogen), and reverse transcription was performed with High capacity cDNA kit (Thermo Fisher Scientific). qPCR was performed using Quant Studio3 (Applied Biosystems) with the following TaqMan probes: Flrt2 (Mm03809571_m1), Flrt3 (Mm01328142 m1), Unc5b (Mm0050454 m1), DCstamp (Mm 04209236 m1), CtsK (Mm00484039 m1), Nfatc1 (Mm00479445 m1), ATP6̄od2 (Mm01222963_m1), 18s (Hs99999901_s1).

\section{In vitro osteoclast differentiation and tartrate-resistant acid phosphatase (TRAP) staining}

BMMs and osteoclasts were prepared as described previously (8). Cells were fixed with $3.7 \%$ formalin and stained using the Acid, Phosphatase, Leukocyte (tartrate-resistant acid phosphatase kit) (387A-1KT, Sigma) according to the manufacturer's instructions. TRAP positive multi-nuclear cells were counted. Recombinant mouse Netrin1 (1109-N1) was purchased from R\&D. Brefeldin A was purchased from Thermo Fisher Scientific.

\section{Retrovirus preparation and transduction}

To prepare retroviral particles, Plat-E packaging cells were transfected with $\mathrm{pMX}$ vectors encoding C-terminally FLAGtagged Flrt2, pSuper vectors encoding siRNA targets for Unc5b (5'-GCCACGCAGATCTACTTCAAGTGTA-3'), and Flrt3 (5'-GA GCACCATTCAGATAACCACTGCA-3') using PEImax (Polysciences). Empty pMX vector and pSuper vector were used as negative controls. After 3 days, medium containing retrovirus was harvested and passed through a syringe filter $(0.45 \mu \mathrm{m}$ pore diameter). BMMs were transduced with retroviruses for $16 \mathrm{~h}$ with hexadimethrine bromide $(8 \mu \mathrm{g} / \mathrm{ml})$ in the presence of M-CSF $(60 \mathrm{ng} / \mathrm{ml})$. Infected cells were selected by culturing for 2 days in the presence of puromycin $(2 \mu \mathrm{g} / \mathrm{ml})$ with M-CSF $(60 \mathrm{ng} / \mathrm{ml})$. Puromycin-resistant BMMs were used for experiments.

\section{Immunoblot analysis}

Cells were lysed with sample buffer $(0.5 \%$ Triton-X $100,20 \mathrm{nM}$ Tris- $\mathrm{HCl}, \mathrm{pH} 7.5,150 \mathrm{mM} \mathrm{NaCl})$. Glycoprotein were pulled down from total cell lysates or culture supernatants using lectin (SIGMA: L1882) to enrich Flrt2. Protein concentrations were determined using the Bradford assay. Equal amounts of lysates were fractionated by SDS-polyacrylamide gel electrophoresis (SDS-PAGE) on a 4-12\% gradient gel and transferred onto polyvinyl difluoride (PVDF) membranes. Western blotting was performed with the following antibodies: anti-Flrt2: AF2877 (R\&D), and anti-Actin: sc-47778 (Santacruz). Rac1 activity was measured using Rac1 activation detection kit (Thermo scientific).

\section{Micro-computed tomography}

Femurs from Flrt $2^{\mathrm{fl} / \mathrm{fl}}$ and Flrt $2^{\mathrm{fl} / \mathrm{fl}} \mathrm{M} \times 1 \mathrm{Cre}$ mice were harvested and scanned by $\mu \mathrm{CT}(\mu \mathrm{CT} 35$, SCANCO Medical AG, Brüttisellen, Switzerland). For trabecular bone analysis, scans were performed at the distal femoral metaphysis $0.4 \mathrm{~mm}$ 
proximal to the growth plate. For cortical bone, the mid-diaphysis of femurs was scanned. All scans were performed at a resolution of $6 \mu \mathrm{m}$ per slice using an X-ray energy of $55 \mathrm{kvp}$ and an integration time of $300 \mathrm{~ms}$. A total of 200 slices for trabecular bone and 50 slices for cortical bone were analyzed using the instrument's software.

\section{Statistical analysis}

All experiments were analyzed using two-way ANOVA or unpaired Student's t-test by Prism 6.0 (GraphPad). P $<0.05$ was considered statistically significant.

\section{ACKNOWLEDGEMENTS}

We thank Dr. Rüdiger Klein for providing Flrt2 ${ }^{\mathrm{fl} / \mathrm{fl}}$ mice. We also thank Dr. Matthew C. Walsh for critical discussion and reading the manuscript. This work was supported in part by NIH grants (AR067726 and AR069546), and The Penn Center for Musculoskeletal Disorders Histology Core (NIH P30AR069619).

\section{CONFLICTS OF INTEREST}

The authors have no conflicting interests.

\section{REFERENCES}

1. Teitelbaum SL and Ross FP (2003) Genetic regulation of osteoclast development and function. Nat Rev Genet 4, 638-649

2. Walsh MC, Kim N, Kadono Y et al (2006) Osteoimmunology: interplay between the immune system and bone metabolism. Annu Rev Immunol 24, 33-63

3. Nakashima T, Hayashi M, Fukunaga T et al (2011) Evidence for osteocyte regulation of bone homeostasis through RANKL expression. Nat Med 17, 1231-1234

4. Xiong J, Onal M, Jilka RL, Weinstein RS, Manolagas SC and O'Brien CA (2011) Matrix-embedded cells control osteoclast formation. Nat Med 17, 1235-1241

5. Moutsopoulos NM, Konkel J, Sarmadi M et al (2014) Defective neutrophil recruitment in leukocyte adhesion deficiency type I disease causes local IL-17-driven inflammatory bone loss. Sci Transl Med 6, 229ra240

6. Souza PP and Lerner UH (2013) The role of cytokines in inflammatory bone loss. Immunol Invest 42, 555-622

7. Miyazaki T, Tokimura F and Tanaka S (2014) A review of denosumab for the treatment of osteoporosis. Patient Prefer Adherence 8, 463-471

8. Kim H, Walsh MC, Takegahara $N$ et al (2017) The purinergic receptor $\mathrm{P} 2 \mathrm{X} 5$ regulates inflammasome activity and hyper-multinucleation of murine osteoclasts. Sci Rep 7, 196

9. Teitelbaum SL (2000) Bone resorption by osteoclasts. Science 289, 1504-1508

10. Yagi M, Miyamoto T, Sawatani $Y$ et al (2005) DC-STAMP is essential for cell-cell fusion in osteoclasts and foreign body giant cells. J Exp Med 202, 345-351

11. Binder NB, Niederreiter B, Hoffmann O et al (2009) Estrogen-dependent and C-C chemokine receptor-2dependent pathways determine osteoclast behavior in osteoporosis. Nat Med 15, 417-424

12. Lacy SE, Bonnemann CG, Buzney EA and Kunkel LM (1999) Identification of FLRT1, FLRT2, and FLRT3: a novel family of transmembrane leucine-rich repeat proteins. Genomics 62, 417-426

13. Karaulanov EE, Bottcher RT and Niehrs C (2006) A role for fibronectin-leucine-rich transmembrane cell-surface proteins in homotypic cell adhesion. EMBO Rep 7, 283-290

14. Egea J, Erlacher C, Montanez E et al (2008) Genetic ablation of FLRT3 reveals a novel morphogenetic function for the anterior visceral endoderm in suppressing mesoderm differentiation. Genes Dev 22, 3349-3362

15. Leyva-Diaz E, del Toro D, Menal MJ et al (2014) FLRT3 is a Robo1-interacting protein that determines Netrin-1 attraction in developing axons. Curr Biol 24, 494-508

16. Maretto S, Muller PS, Aricescu AR, Cho KW, Bikoff EK and Robertson EJ (2008) Ventral closure, headfold fusion and definitive endoderm migration defects in mouse embryos lacking the fibronectin leucine-rich transmembrane protein FLRT3. Dev Biol 318, 184-193

17. Muller PS, Schulz R, Maretto S et al (2011) The fibronectin leucine-rich repeat transmembrane protein Flrt2 is required in the epicardium to promote heart morphogenesis. Development 138, 1297-1308

18. O'Sullivan ML, de Wit J, Savas JN et al (2012) FLRT proteins are endogenous latrophilin ligands and regulate excitatory synapse development. Neuron 73, 903-910

19. Yamagishi S, Hampel F, Hata K et al (2011) FLRT2 and FLRT3 act as repulsive guidance cues for Unc5-positive neurons. EMBO J 30, 2920-2933

20. Tai-Nagara I, Yoshikawa Y, Numata N et al (2017) Placental labyrinth formation in mice requires endothelial FLRT2/UNC5B signaling. Development 144, 2392-2401

21. Seiradake E, del Toro D, Nagel D et al (2014) FLRT structure: balancing repulsion and cell adhesion in cortical and vascular development. Neuron 84, 370-385

22. Jackson VA, del Toro D, Carrasquero $M$ et al (2015) Structural basis of latrophilin-FLRT interaction. Structure $23,774-781$

23. Maruyama K, Kawasaki T, Hamaguchi M et al (2016) Bone-protective functions of netrin 1 Protein. J Biol Chem 291, 23854-23868

24. Seiradake E, Jones EY and Klein R (2016) Structural Perspectives on Axon Guidance. Annu Rev Cell Dev Biol $32,577-608$

25. Zhu S, Zhu J, Zhen G et al (2019) Subchondral bone osteoclasts induce sensory innervation and osteoarthritis pain. J Clin Invest 129, 1076-1093

26. Takegahara N, Kim H, Mizuno H et al (2016) Involvement of receptor activator of nuclear factor-kappaB ligand (RANKL)-induced incomplete cytokinesis in the polyploidization of osteoclasts. J Biol Chem 291, 3439-3454

27. Paiva KBS and Granjeiro JM (2017) matrix metalloproteinases in bone resorption, remodeling, and repair. Prog Mol Biol Transl Sci 148, 203-303 